\title{
The role of VirA and VirG phosphorylation in chemotaxis towards acetosyringone by Agrobacterium tumefaciens
}

\author{
Adrian C. V. Palmer and Charles H. Shaw* \\ Department of Biological Sciences, University of Durham, South Road, Durham DHI 3LE, UK
}

(Received 11 May 1992; revised 14 August 1992; accepted I September 1992)

\begin{abstract}
The Ti-plasmid-encoded two-component sensor-regulator system comprising VirA and VirG confers upon Agrobacterium tumefaciens the ability to respond chemotactically to nanomolar concentrations of vir-inducing phenolics such as acetosyringone. Non-phosphorylatable, mutant VirA and VirG proteins are incapable of replacing their wild-type counterparts in conferring this phenotype. This indicates that, like vir-gene induction in response to acetosyringone, chemotaxis to the same ligand involves phosphorylation of VirA and VirG. However, unlike vir-induction, deletion of the periplasmic domain of VirA severely curtails acetosyringone chemotaxis, suggesting that acetosyringone may mediate effects through more than one region of VirA. When introduced into strains expressing wild-type VirA and VirG, the non-phosphorylatable versions suppress chemotaxis towards acetosyringone, implying that mutant copies of VirA and VirG compete with their wild-type counterparts in interactions between VirA and VirG.
\end{abstract}

\section{Introduction}

Environmental sensing is of particular importance to Agrobacterium tumefaciens (Shaw, 1991). The bacterium's existence in the rhizosphere, coupled with a requirement to interact with wounded plants, has led to the evolution of a highly sensitive chemotaxis system (Ashby et al., 1987, 1988; Loake et al., 1988). Motility is important in rhizosphere competence (Shaw et al., 1991) and chemotaxis towards plant exudates is a component of virulence (Hawes \& Smith, 1989; Hawes et al., 1988). The most intense chemotactic response for avirulent, Ti-plasmid-cured $A$. tumefaciens is towards micromolar levels of plant sugars (Loake et al., 1988). The Ti-plasmid confers the additional ability to respond specifically to nanomolar amounts of wound-exuded phenolic compounds, such as acetosyringone (Ashby et al., 1987, 1988). This property requires the Ti-plasmid virulence genes virA and virG (Shaw et al., 1988). At higher acetosyringone concentrations, VirA and VirG mediate a second response, the induction of the other vir-operons, products of which act in concert to transfer T-DNA to the plant cell (Howard \& Citovsky, 1990).

VirA is a $92 \mathrm{kDa}$ transmembrane protein (Winans et al., 1989), and forms the sensor part of a two-component system with the $30 \mathrm{kDa}$ cytoplasmic transcriptional

* Author for correspondence. Tel. (091) 3742430 ; fax (091) 3742417. regulator, VirG (Winans et al., 1986). Phenolics are believed to interact with the second transmembrane (TM2) domain of VirA (Melchers et al., 1989). The C-terminal cytoplasmic domain of VirA can be autophosphorylated, at histidine-474 (Huang et al., 1990b; Jin et al., 1990b), and can transfer this phosphate to an aspartate residue (D52) in VirG (Jin et al., 1990a,b). Through its C-terminal domain (Powell \& Kado, 1990), VirG binds specifically to the vir-box (Jin et al., 1990c; Pazour \& Das, 1990b; Powell et al., 1989), a conserved regulatory element found upstream of most of the virgenes. Non-phosphorylatable mutant VirA and VirG proteins fail to induce vir-gene expression, suggesting that this system behaves in a similar manner to other two-component regulators such as $\mathrm{NtrB} / \mathrm{C}$ (Jin et al., $1990 a, b, c)$. Indeed, there is considerable scope for interactions between different two-component systems, such as between VirG and PhoB (Aoyama et al., 1991). However, nothing is known of how $\operatorname{Vir} A / G$ can mediate two responses to wound phenolics (Shaw et al., 1988): chemotaxis at low concentrations $\left(10^{-7} \mathrm{M}\right)$; and virinduction at higher ligand concentrations $\left(10^{-5} \mathrm{M}\right)$.

VirA is involved in additional interactions. Sugars, e.g. galactose and glucose, can also enhance vir-induction (Cangelosi et al., 1990; Huang et al., 1990a). This requires the products of $c h v E$, a chromosomal gene encoding a homologue of the Escherichia coli periplasmic ribose/galactose binding protein, and $\operatorname{vir} A$ and $\operatorname{vir} G$ 
Table 1. Bacterial strains and plasmids used

\begin{tabular}{|c|c|c|}
\hline Strain & Characteristics* & Reference \\
\hline $\begin{array}{l}\text { A. tumefaciens } \mathrm{C} 58 \mathrm{Cl} \\
\text { Ery } \mathrm{Cm}\end{array}$ & Ery ${ }^{r}, \mathrm{Cm}^{r}$ Ti-plasmid-cured & Van Larebeke et al. (1974) \\
\hline E. coli $\mathrm{C} 2110$ & polA, rha, his, $\mathrm{Nal}^{\mathrm{r}}$ & Prince \& Borlam (1985) \\
\hline Plasmid & Characteristics & Reference \\
\hline $\begin{array}{l}\text { pVK219 } \\
\text { pVK225 } \\
\text { pVK257 } \\
\text { pSG687 } \\
\text { pSG689 } \\
\text { pTB108 } \\
\text { pRS0824 } \\
\text { pIB100 } \\
\text { pIB415 }\end{array}$ & 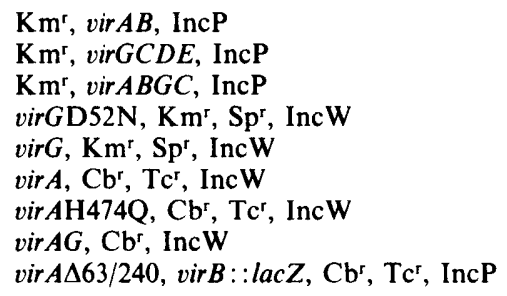 & $\begin{array}{l}\text { Knauf \& Nester (1982) } \\
\text { Jin et al. (1990a) } \\
\text { Jin et al. (1990b) } \\
\text { Ankenbauer et al. (1991) }\end{array}$ \\
\hline
\end{tabular}

* Nal, nalidixic acid; $\mathrm{Cb}$, carbenicillin; Ery; erythromycin; $\mathrm{Km}$, kanamycin; Sp, spectinomycin; r, resistant.

(Cangelosi et al., 1990; Huang et al., 1990a). Deletion of the periplasmic domain of VirA abolishes this effect, suggesting that $\mathrm{ChvE}$ interacts with this region (Cangelosi et al., 1990). This domain contains a sequence conserved with the methyl-accepting chemotaxis protein $\operatorname{Trg}$ (Cangelosi et al., 1990) which in E. coli interacts with the ribose- and galactose-binding proteins (Hazelbauer et al., 1990). Interestingly, chvE mutants are defective in sugar chemotaxis (Cangelosi et al., 1990). The periplasmic domain of VirA also appears to be involved in pH sensing (Melchers et al., 1989).

VirA is clearly a multifunctional protein, but exactly how it interacts with VirG and the cytoplasmic signalling pathway to mediate chemotaxis towards phenolics is unclear. In this paper, we report data from experiments designed to investigate the role of $\operatorname{Vir} A / G$ phosphorylation in chemotaxis towards acetosyringone.

\section{Methods}

Bacterial strains and plasmids. Bacterial strains and plasmids used in this work are listed in Table 1.

Media and chemicals. E. coli and $A$. tumefaciens were grown in LAB $M$ nutrient broth number 2 (Amersham). A. tumefaciens was also cultured in Min A + glucose (Miller, 1972). Chemotaxis medium consisted of $0.1 \mathrm{~mm}$-EDTA, $\mathrm{pH} 7.0$ and $10 \mathrm{~mm}-\mathrm{KH}_{2} \mathrm{PO}_{4}, \mathrm{pH} 7.0$ (Adler, 1973). Swarm plates were prepared from nutrient broth supplemented with $0.17 \%$ Bacteriological agar Number 1 (Oxoid). Antibiotics (Sigma) were used as previously described (Leemans et al., 1981). All other reagents were from BDH, unless otherwise stated.

Preparation of plasmids. Plasmids pSG687, pSG689, pIB100, pIB415, pTB 108 and pRS0824 were purified from $100 \mathrm{ml}$ overnight cultures of E. coli C2110 using Qiagen plasmid midi columns (Diagen).

Preparation of strains. Plasmids carrying copies of virA and virG were electroporated into $A$. tumefaciens backgrounds carrying cosmid clones. Cultures $(5 \mathrm{ml})$ of the recipient strain were grown overnight with antibiotic selection. Two successive $1.5 \mathrm{ml}$ cell samples were pelleted in the same Eppendorf tube, washed three times in $1 \mathrm{ml}$ ice-cold $1 \mathrm{~mm}$ HEPES buffer, $\mathrm{pH} 7 \cdot 0$, and once in $1 \mathrm{ml}$ chilled sterile $10 \%(\mathrm{v} / \mathrm{v})$ glycerol. The cells were resuspended in $40 \mu \mathrm{l} 10 \%(\mathrm{v} / \mathrm{v})$ glycerol $\left(4^{\circ} \mathrm{C}\right)$ and $3 \mu \mathrm{l}$ plasmid DNA, prepared by the alkaline lysis 'miniprep' method (Sambrook et al., 1989) was mixed in. The mixture was transferred to an ice-cold $0 \cdot 2 \mathrm{~cm}$ electroporation cuvette (Bio-Rad) and subjected to a $2.5 \mathrm{kV}$ pulse dissipated at $200 \Omega, 25 \mu \mathrm{F}$ using a Gene Pulser (Bio-Rad). Successful transformations were associated with a time constant in the order of $4.7 \mathrm{~ms}$. Immediately after transformation, $1 \mathrm{ml}$ nutrient broth was added to the cuvette and the cells incubated with shaking at $27^{\circ} \mathrm{C}$ for $2 \mathrm{~h}$. Following pelleting and resuspension in $100 \mu \mathrm{l}$ nutrient broth the cells were plated out and grown with appropriate antibiotic selection. Plasmid presence was verified by restriction pattern analysis of DNA prepared by the alkaline lysis 'miniprep' method. Motile recombinant populations were selected by three sequential rounds of swarming on selective plates (Loake et al., 1988). Culture purity was confirmed microscopically using a Nikon Optiphot microscope equipped with phase-contrast optics and by a plate test for the production of 3-keto-lactose (Bernaerts \& De Ley, 1963).

Preparation of attractants. Stock solutions of acetosyringone (Janssen Chimica) were prepared in decimal concentration decrements from $10^{-2}$ to $10^{-5} \mathrm{M}$ using ethyl acetate:methanol $(1: 1)$ as a solvent. Solutions $\left(10^{-5}\right.$ to $\left.10^{-8} \mathrm{M}\right)$ were prepared for assays by dilution of these stocks with 1000 vols of chemotaxis medium. This ensured a constant and low concentration of solvent in all assays. Control solutions consisted of chemotaxis medium with an equal concentration of solvent to that in assay solutions, but no acetosyringone.

Chemotaxis assays. Cultures $(50 \mathrm{ml})$ of the strains to be assayed were grown for $16 \mathrm{~h}$ to exponential phase in Min A + glucose. Samples $(40 \mathrm{ml})$ were pelleted at $7000 \mathrm{~g}$ for $10 \mathrm{~min}$ in sterile tubes. The pellets were resuspended and washed in $40 \mathrm{ml}$ chemotaxis medium. This was repeated, concentrating the pellets in $30,20,15$ and finally $10 \mathrm{ml}$ of chemotaxis medium. Blindwell assays (Shaw et al., 1988) were performed using $200 \mu \mathrm{l}$ of cell suspension in Boyden chambers (Armitage et al., 1977). Nuclepore $13 \mathrm{~mm}$ diameter polycarbonate filters with $8 \mu \mathrm{m}$ pores (Sterilin) were used in assay chambers. After $2 \mathrm{~h}$ inversion the number of cells passing through the membranes was counted using a Coulter Electronics Multisizer II, fitted with a $30 \mu \mathrm{m}$ aperture (Shaw et al., 1988). The density of the culture initially 
introduced into the chambers was also ascertained. Control values were obtained from parallel assays using chemotaxis medium as attractant. All assays were repeated in triplicate for each strain and attractant concentration. Three separate Multisizer readings were taken for each assay.

Presentation of results. Results were presented in the form of the Chemotactic Index (CI) which is a measure of the proportion of cells in the bacterial population attracted towards the attractant. The $\mathrm{CI}$ corrects for differing initial cell density, and arises from the observation that for a given strain, the proportion of cells attracted to a particular attractant concentration is a constant, over the range of cell densities used in the experiments described in this paper (data not shown). To calculate the $\mathrm{CI}$, Multisizer readings were converted to numbers of cells per $\mathrm{ml}$. Control values were subtracted from each point to give a figure for number of cells attracted solely by the acetosyringone. To correct for differences in original culture density, the data were then divided by the cell density of the suspension introduced into the chambers. Averaged figures were then converted to percentages of cells attracted by the compound (the $\mathrm{CI}$ ) and graphically presented. The $\mathrm{CI}$ is thus a reproducible value, and permits comparison from one experiment to the next. In an average experiment, a $\mathrm{CI}$ of 2 represents approximately $10^{7}$ bacteria per $\mathrm{ml}$ in the attractant chamber.

$$
\mathrm{CI}=\frac{[\text { Cells in upper chamber }]-[\text { Cells in control assay upper chamber }]}{[\text { Cells initially introduced to lower chamber }]} \times 100 \%
$$

\section{Results}

Recently, a number of site-specific mutations in $\operatorname{vir} A$ and virG (see Table 1) have been constructed and made available by the group of Prof. E. W. Nester (Seattle) (Cangelosi et al., 1990; Jin et al., 1990a, b). pSG687 and pRS0824 encode mutant VirG and VirA proteins, respectively, in which the phosphorylated residue has been altered. Neither protein can mediate vir-induction (Jin et al., 1990a,b). pIB415 expresses a mutant VirA protein, in which residues 63-240 (essentially the whole periplasmic domain) have been deleted. This mutant is still capable of mediating vir-induction (Cangelosi et al., 1990). pTB108 (Jin et al., 1990b) and pSG689 (Jin et al., $1990 a$ ) express wild-type VirA and VirG, respectively.

To investigate the pathway effecting acetosyringone chemotaxis, the ability of these mutant proteins to confer this phenotype upon $A$. tumefaciens was investigated. Due to plasmid incompatibility, it was necessary to analyse the wild-type and mutated virA and virG (on IncW plasmids) in $A$. tumefaciens backgrounds carrying vir-gene combinations on the IncP cosmids pVK219 (virAB) pVK225 (virGCDE) and pVK257 (virABGC) (Knauf \& Nester, 1982). Plasmids were introduced into these backgrounds by electroporation (see Methods). Presence of both plasmids in each strain was confirmed by restriction pattern analysis of DNA purified by miniprep (see Methods). Motile populations of bacteria were then selected by swarm plate, and assayed for chemotaxis by Blindwell assay against several acetosyringone concentrations (see Methods).

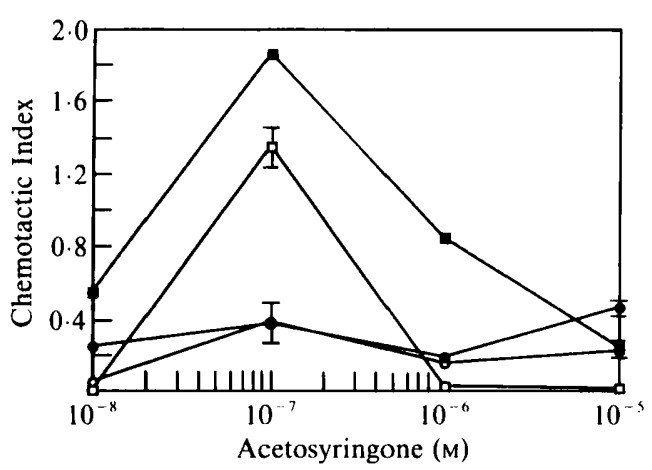

Fig. 1. Response curves showing chemotaxis towards varying concentrations of acetosyringone by $\boldsymbol{A}$. tumefaciens derivatives harbouring either $\square$, pVK257 (virABGC); O, pVK219 (virAB); •, pVK219 and pSG687 (virGD52N); or $\square$, pVK 219 and pSG689 (virG). Blindwell assays and data processing as in Methods. Error bars indicate one standard deviation from the mean.

\section{The requirement for VirG phosphorylation in mediating chemotaxis}

Fig. 1 depicts the results of acetosyringone chemotaxis experiments, using $A$. tumefaciens harbouring either pVK219 (virAB) or pVK257 (virABGC) alone, and strains harbouring both pVK219 and pSG687 (virGD52N) or pVK219 and pSG689 (virG). In agreement with our previous results (Shaw et al., 1988), pVK257 can confer chemotaxis to acetosyringone, with a peak of attraction at $10^{-7} \mathrm{M}$. Similar results were obtained from strains harbouring pIB 100 , which carries wild-type $\operatorname{vir} A$ and $\operatorname{vir} G$ only (data not shown). The fact that pVK219-harbouring cells lack this response, shows that virA alone is insufficient to mediate chemotaxis to acetosyringone. However, the introduction into pVK219-harbouring cells of pSG687 demonstrates that the non-phosphorylatable mutant of VirG, is not able to complement this deficit, when compared to the wild-type VirG encoded by pSG689. This indicates that phosphorylation of VirG is necessary for acetosyringone chemotaxis.

\section{The requirement for VirA phosphorylation in mediating chemotaxis}

Fig. 2 illustrates chemotaxis assays in which the test strains all harboured pVK225 (virGCDE) either alone, or in combination with pRS0824 (virAH474Q), or pTB108 (virA). pVK257 data from Fig. 1 is shown by way of comparison. In accordance with our previous results (Shaw et al., 1988), pVK225 is incapable of conferring chemotaxis towards acetosyringone, demonstrating that vir $G$ alone is insufficient for this response. Addition of the non-phosphorylatable VirA (encoded by pRS0824) 


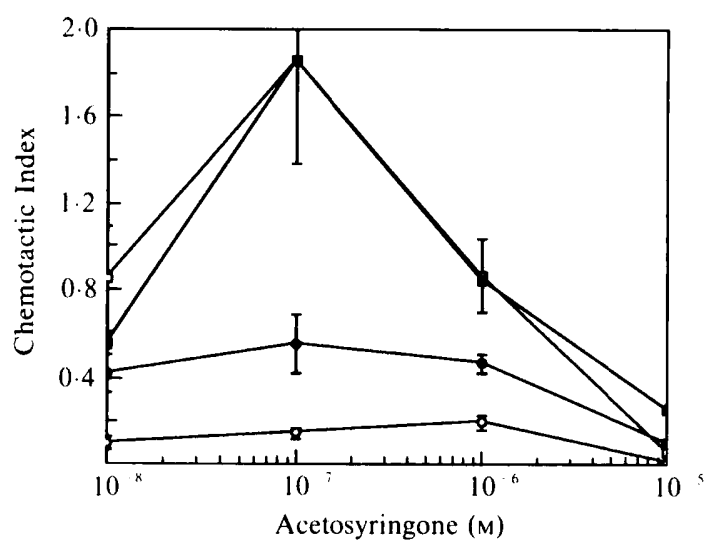

Fig. 2. Response curves showing chemotaxis towards varying concentrations of acetosyringone by $A$. tumefaciens derivatives harbouring either 1 , pVK 257 (virABGC); O, pVK225 (virGCDE); •, pVK225 and pRS0824 (virAH474Q); or $\square$, pVK225 and pTB108 (virA). Blindwell assays and data processing as in Methods. Error bars indicate one standard deviation from the mean.

provides very marginal chemotactic competency compared to the wild-type situation conferred by pVK 257 or pVK225 and pTB108 in combination. The non-phosphorylatable derivative of $\operatorname{vir} A$ is thus incapable of complementing for the wild-type protein, suggesting that phosphorylation of VirA is required for acetosyringone chemotaxis.

\section{The role of the periplasmic region of $\operatorname{Vir} A$}

In Fig. 3, the effect of the periplasmic deletion of vir $A$ upon chemotaxis is shown in comparison with the data for pVK257 from Fig. 1. pIB415 (vir $A \Delta 63 / 240$ ) which encodes a truncated version of $\operatorname{VirA}$, missing the periplasmic portion of the protein, is able only partially to restore chemotactic ability in concert with wild-type vir $G$ carried by pSG689. The peak is substantially smaller and shifted to a higher concentration, relative to the wild-type situation. This suggests that while the periplasmic domain of VirA is non-essential for virinduction by acetosyringone, it is important in acetosyringone chemotaxis.

\section{The interaction between wild-type and mutant} two-component regulatory system molecules

From Fig. 4 it is apparent that the addition of mutant copies of VirA and VirG (encoded by pRS0824 and pSG687, respectively) to a background harbouring their wild-type counterparts (encoded by pVK257) decreases chemotactic competency. Using half-logarithmic increments, it appears that mutant copies of VirA and VirG in a wild-type background cause a shift in the

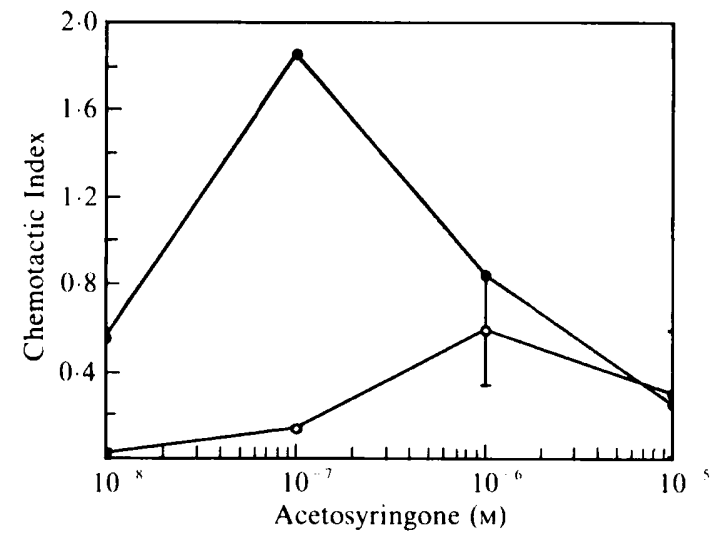

Fig. 3. Response curves showing chemotaxis towards varying concentrations of acetosyringone by $A$. tumefaciens derivatives harbouring either - pVK257 (virABGC); or O, pSG689 (virG) and pIB415 (virA $\triangle 63 / 240)$. Blindwell assays and data processing as in Methods. Error bars indicate one standard deviation from the mean.

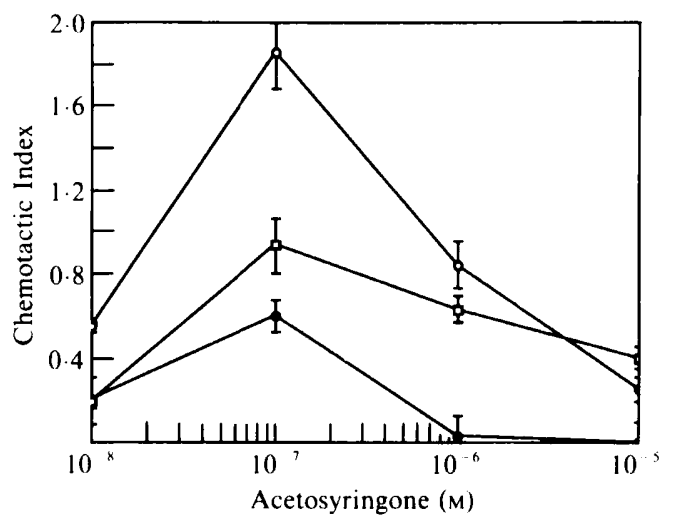

Fig. 4. Response curves showing chemotaxis towards varying concentrations of acetosyringone by $A$. tumefaciens derivatives harbouring either O, pVK257 (virABGC); $\bullet$, pVK257 and pSG687 (virGD52N); or $\square$, pVK257 and pRS0824 (virAH474Q). Blindwell assays and data processing as in Methods. Error bars indicate one standard deviation from the mean.

chemotaxis optimum towards higher attractant concentrations (data not shown). As the vir-genes on these plasmids are expressed from their normal promoters, it is to be expected that wild-type and mutant proteins would be transcribed at equivalent levels. This suggests that the mutant copies of VirA and VirG interfere with normal signalling in a wild-type background.

\section{Discussion}

The non-phosphorylatable versions of VirA and VirG proteins are not competent to mediate chemotaxis towards acetosyringone. This deficiency when compared to their wild-type counterparts implies a requirement for phosphorylation if chemotaxis is to occur efficiently. The 
implicit suggestion that phospho-VirA and phosphoVirG are required for chemotaxis is consistent with the previously reported phosphorylation of VirG by phospho-VirA during the induction of vir-gene expression (Jin et al., 1990a,b). It would appear that VirA, the sensor, is capable of sensing a broad band of acetosyringone concentrations, and effecting two responses, depending upon the sensed concentration. PhosphoVirG is the active, stable, transcriptional activator, capable of sequence-specific, high-affinity DNA binding (Jin et al., $1990 a, c$; Pazour \& Das, $1990 a, b$; Powell et al., 1989; Powell \& Kado, 1990). How it can fulfil this role at high concentrations of acetosyringone and mediate chemotaxis at lower concentrations is not yet clear.

A possible explanation for the ability of VirA and VirG to mediate two different effects, in response to differing concentrations of the ligand would be that phospho-VirG has a high affinity for a component of the cytoplasmic chemotaxis signalling pathway (possibly a CheA homologue) and a somewhat lower affinity for virbox DNA. Thus at low concentrations of the ligand ( $\ll 1$ molecule per receptor) the small amounts of phospho-VirG produced would preferentially interact with the chemotaxis pathway. At higher concentrations ( $>10$ molecules per receptor) sufficient phospho-VirG is produced to participate in significant vir-box binding, and hence vir-induction.

The periplasmic region of VirA has been shown to be essential for vir-induction by sugars through the ChvE pathway (Cangelosi et al., 1990). It has also been shown not to be involved in chemotaxis towards sugars, where a mechanism involving ChvE and a putative Trg analogue are thought to effect the response. The role of this region of the molecule in the cell's response to acetosyringone is unclear. The putative acetosyringone binding site of VirA is thought to be associated with the transmembrane regions of the molecule (Cangelosi et al., 1990; Melchers et al., 1989). Deletion of the periplasmic region is reported to have a variety of effects on vir-induction, with one report of no change (Melchers et al., 1989) and another describing increasing sensitivity of response (Cangelosi et al., 1990). The reported increased sensitivity to acetosyringone is thought to result from the molecule adopting a non-wild-type conformation in the membrane. The periplasmic deletion appears to severely curtail chemotaxis towards acetosyringone, which may be simply an effect of the altered conformation. However, the data does indicate that the periplasmic domain, while inessential for vir-induction, is required for chemotaxis. This suggests that VirA may possess more than one site with differing affinities for acetosyringone. It is also possible that acetosyringone does not interact directly with VirA, but rather with one or more binding proteins in the periplasm, with different ligand affinities. Differential concentration effects would then be achievable by these proteins interacting with different domains of VirA.

The addition of mutant copies of VirA and VirG to the wild-type chemotaxis system encoded by pVK 257 decrease its effectiveness. The ability to respond to the stimulus is reduced, with a shift in the chemotaxis peak towards higher ligand concentrations. In the case of vir $A$ mutants, it is hypothesized that of the relatively small number of acetosyringone molecules interacting with cell surfaces, a proportion interact with mutant molecules to no effect and a proportion elicit a chemotaxis-inducing signal through the wild type. This decreases the number of cells attracted. The explanation of the results following the addition of mutant virG is thought to be that the cell's ability to respond to acetosyringone is depleted because its proportion of competent signal intermediates is lessened. Mutant non-phoshorylatable VirG molecules compete with wild-type intermediates for association with VirA, meaning that although acetosyringone presence is detected by VirA, the chemotactic signal is transmitted at lower levels.

\footnotetext{
The authors would like to thank their colleagues in the Playgroup for making science fun, and Prof. E. W. Nester for making virA and virG clones available. A.C.V.P. is supported by a studentship from the North of England Children's Cancer Research Fund.
}

\section{References}

AdLer, J. (1973). A method for measuring chemotaxis and use of the method to determine optimum conditions for chemotaxis by Escherichia coli. Journal of General Microbiology 74, 77-91.

Ankenbauer, R. G., Best, E. A., Palanca, C. A. \& Nester, E. W. (1991). Mutants of the Agrobacterium tumefaciens virA gene exhibiting acetosyringone-independent expression of the vir regulon. Molecular Plant-Microbe Interactions 4, 400-406.

aoyama, T., Takanami, M., Makino, K. \& OKa, A. (1991). Cross-talk between the virulence and phosphate regulons of Agrobacterium tumefaciens caused by an unusual interaction of the transcriptional activator with a regulatory DNA element. Molecular and General Genetics 227, 385-390.

Armitage, J. P., Josey, D. P. \& Smith, D. G. (1977). A simple qualitative method for measuring chemotaxis and motility in bacteria. Journal of General Microbiology 102, 199-202.

Ashby, A. M., Watson, M. D. \& Shaw, C. H. (1987). A Ti-plasmid determined function is responsible for chemotaxis of Agrobacterium tumefaciens towards the plant wound product acetosyringone. FEMS Microbiology Letters 41, 189-192.

Ashby, A. M., Watson, M. D., Loake, G. J. \& Shaw, C. H. (1988). Ti-plasmid-specified chemotaxis of Agrobacterium tumefaciens $\mathrm{C} 58 \mathrm{Cl}$ toward vir-inducing phenolic compounds and soluble factors from monocotyledonous and dicotyledonous plants. Journal of Bacteriology 170, 4181-4187.

Bernaerts, M. J. \& De Ley, J. (1963). A biochemical test for Crown Gall. Nature, London 197, 406-407.

Cangelosi, G. A., Ankenbauer, R. G. \& Nester, E. W. (1990). Sugars induce the Agrobacterium tumefaciens virulence genes via a periplasmic binding protein and the virA protein. Proceedings of the National Academy of Sciences of the United States of America 87, $6708-6712$. 
Hawes, M. C. \& SMITH, L. Y. (1989). Requirement for chemotaxis in pathogenicity of Agrobacterium tumefaciens on roots of soil grown pea plants. Journal of Bacteriology 171, 5668-5671.

Hawes, M. C., SMITH, L. Y. \& HowarTh, A. J. (1988). Agrobacterium tumefaciens mutants deficient in chemotaxis to root exudates: Molecular Plant-Microbe Interactions 1, 182-186.

Hazelbauer, G. L., Yaghmal, R., Burrows, G. G., Baumgartner, J. W., Dutton, D. P. \& Morgan, D. G. (1990). Transducers: transmembrane receptor proteins involved in bacterial chemotaxis. In Biology of the Chemotactic Response, pp. 107-134. Edited by J. P. Armitage \& J. M. Lackie. Cambridge: Cambridge University Press.

Howard, E. \& Citovsky, V. (1990). The emerging structure of the Agrobacterium T-DNA transfer complex. BioEssays 12, 103-108.

Huang, M. L. W., Cangelosi, G. A., Halperin, W. \& Nester, E. W. (1990a). A chromosomal Agrobacterium tumefaciens gene required for effective plant signal transduction. Journal of Bacteriology 172 , 1814-1822.

Huang, Y., Morel, P., Powell, B. \& Kado, C. I. (1990b). virA, a coregulator of Ti-specified virulence genes is phosphorylated in vitro. Journal of Bacteriology 172, 1142-1144.

Jin, S., Prusti, R. K., Roitsch, T., Ankenbauer, R. G. \& Nester, E. W. $(1990 a)$. Phosphorylation of the VirG protein of Agrobacterium tumefaciens by the autophosphorylated VirA protein: essential role in biological activity of VirG. Journal of Bacteriology $172,4945-4950$.

Jin, S., Roitsch, T., Ankenbauer, R. G., Gordon, M. P. \& Nester, E. W. (1990b). The virA protein of Agrobacterium tumefaciens is autophosphorylated and is essential for vir gene regulation. Journal of Bacteriology 172, 525-530.

Jin, S., Roitsch, T., Christie, P. J. \& Nester, E. W. (1990c). The regulatory vir $G$ protein specifically binds to a cis-acting regulatory sequence involved in transcriptional activation of Agrobacterium tumefaciens virulence genes. Journal of Bacteriology 172, 531-537.

KNAUF, V. C. \& Nester, E. W. (1982). Wide host range cloning vectors: a cosmid clone bank of an Agrobacterium Ti-plasmid. Plasmid 8, 45-54.

Leemans, J., Shaw, C. H., Deblaere, R., De Greve, H., Hernalsteens, J.-P., Van Montagu, M. \& Schell, J. (1981). Sitespecific mutagenesis of Agrobacterium Ti-plasmids and transfer of genes to plant cells. Journal of Molecular and Applied Genetics 1, $149-164$.

Loake, G. J., Ashby, A. M. \& Shaw, C. H. (1988). Attraction of Agrobacterium tumefaciens $\mathrm{C} 58 \mathrm{Cl}$ towards sugars involves a highly sensitive chemotaxis system. Journal of General Microbiology 134, $1427-1432$

Melchers, L. S., Regensburg-Tuink, T. J. G., Bourret, R. B., Sedee, N. J. A., Schilperoort, R. A. \& HooykaAs, P. J. J. (1989).
Membrane topology and functional analysis of the sensory protein virA of Agrobacterium tumefaciens. EMBO Journal 8, 1919-1925.

Miller, J. H. (1972). Experiments in Molecular Genetics. New York: Cold Spring Harbor Laboratory.

Pazour, G. J. \& DAS, A. $(1990 a)$. Characterisation of the VirG binding site of Agrobacterium tumefaciens. Nucleic Acids Research 18, 6909-6913.

Pazour, G. J. \& Das, A. (1990b). virG, an Agrobacterium tumefaciens transcriptional activator initiates translation at a UUG codon and is a sequence-specific DNA-binding protein. Journal of Bacteriology 172, 1241-1249.

Powell, B., Rogowsky, P. \& Kado, C. I. (1989). virG of Agrobacterium tumefaciens plasmid pTiC58 encodes a DNA-binding protein. Molecular Microbiology 3, 411-419.

Powell, B. S. \& KADO, C. I. (1990). Specific binding of VirG to the vir box requires a $\mathrm{C}$-terminal domain and exhibits a minimum concentration threshold. Molecular Microbiology 4, 2159-2165.

Prince, A. S. \& BorLAM, T. (1985). Isolation of a DNA fragment containing replication functions from IncP2 megaplasmid pMG2. Journal of Bacteriology 161, 792-794.

Sambrook, J., Fritsch, E. F. \& Maniatis, T. (1989). Molecular Cloning: a Laboratory Manual. Cold Spring Harbor: Cold Spring Harbor Laboratory Press.

SHaw, C. H. (1991). Swimming against the tide: chemotaxis in Agrobacterium. BioEssays 13, 25-29.

Shaw, C. H., Ashby, A. M., Brown, A. P., Royal, C., Loake, G. J. \& SHAW, C. H. (1988). vir $A$ and $G$ are the Ti-plasmid functions required for chemotaxis of Agrobacterium tumefaciens toward acetosyringone. Molecular Microbiology 2, 413-418.

Shaw, C. H., Loake, G. J., Brown, A. P., Garrett, C. S., Deakin, W., Alton, G., Hall, M., Jones, S. A., O'Leary, M. \& Primavesi, L. (1991). Isolation and characterization of behavioural mutants and genes from Agrobacterium tumefaciens. Journal of General Microbiology 137, 1939-1953.

Van Larebeke, N., Engler, G., Holsters, M., Van Den Elsacker, S., Zaenen, I., SChILPeroort, R. A. \& Schell, J. (1974). Large plasmid in Agrobacterium tumefaciens essential for crown gall inducing ability. Nature, London 252, 169-170.

Winans, S. C., Ebert, P. R., Stachel, S. E., Gordon, M. P. \& NESTER, E. W. (1986). A gene essential for Agrobacterium virulence is homologous to a family of positive regulatory loci. Proceedings of the National Academy of Sciences of the United States of America 83, 8278-8282.

Winans, S. C., Kerstetter, R. A., Ward, J. E. \& Nester, E. W. (1989). A protein required for transcriptional regulation of Agrobacterium virulence genes spans the cytoplasmic membrane. Journal of Bacteriology 171, 1616-1622. 